\title{
Editorial
}

\section{Genitourinary Syndrome of Menopause and Its Management}

The age of menopause has remained practically unchanged over the centuries. However, the longevity of women is relatively recent with rapid decline in maternal mortality and medical advances in the last century. In the present times, women expect to spend one-third of their lives beyond menopause. This indeed is the justification of the physician's interest in the health of the adult women.

The STRAW + 10 staging system for reproductive aging in women assists the healthcare providers in understanding and classifying the menopausal transition. ${ }^{1}$ Vasomotor symptoms (VMSs) are the commonest complaints in the perimenopause and first 1 to 2 years postmenopause. Majority of women report spontaneous relief of VMS after this period. In the late postmenopause period beyond 5 to 6 years, progressive increase in genitourinary symptoms due to urogenital atrophy related to estrogen deficiency becomes more prevalent. These include vulvovaginal pruritis, dryness, burning and irritation. In addition, urinary complaints, like urgency, frequency, dysuria and recurrent urinary tract infections (UTIs), are often reported. The symptom severity varies affecting almost $50 \%$ of women beyond 50 years of age and often results in sexual dysfunction. Majority of women are unaware that these chronic genitourinary symptoms are directly related to estrogen deficiency. ${ }^{2}$

The traditional term vulvovaginal atrophy (VVA) only describes the appearance and does not include lower urinary tract. Similarly, the term 'atrophic vaginitis' implies infection or inflammation of the vagina, which is indeed a misnomer. Therefore, in the terminology consensus conference in May 2013 held by the North American Menopause Society (NAMS) and International Society for the Study of Women's Sexual Health (ISSWSH) agreed on the term 'Genitourinary Syndrome of Menopause' (GSM) for this condition. This was subsequently endorsed in 2014 by the two boards. ${ }^{3}$

In contrast to vasomotor symptoms that generally settle over time even without interventions, GSM is usually progressive and unlikely to resolve without treatment. It can indeed have a major effect on quality of life (QOL) in general and a woman's sexual health in particular. A thorough history to identify contributing factors and exclusion of other causes is essential. A complete pelvic examination is required to exclude other pathologic conditions that may cause similar symptom and confirm signs of GSM. These include decreased moisture and elasticity, resorption of labia minora, loss of vaginal rugae and hymenal remnants, uterovaginal prolapse (UV) prolapse and prominence of urethral meatus. Effective therapeutic interventions can then be initiated.

First-line therapeutic options for women with symptomatic mild GSM include nonhormonal lubricants, like KY gel or regular use of long-acting vaginal moisturizers, like Replens, available over the counter. In women with moderate to severe symptoms, low dose vaginal estrogen therapy or less commonly systemic hormone replacement therapy (HRT) is advised. Therapy with estrogen creams or tablets needs to be individualized. Initially, daily vaginal use for 2 weeks can be followed by a maintenance frequency of 2 to 3 doses per week. The lowest effective dose and frequency of vaginal estrogen providing the desired effect is recommended. Application should be at least 12 hours before coital activity to prevent estrogen absorption by the partner. Routine endometrial surveillance in asymptomatic women using vaginal estrogen therapy (ET) is not recommended. However, endometrial safety data for use beyond 1 year is not available.

Ospemifene, a unique selective estrogen receptor modulator (SERM), is another option for dyspareunia. In women taking $60 \mathrm{mg}$ ospemifene orally daily, VMS were reported by 7\% women only. A progestogen is not required when low-dose vaginal estrogen or ospemifene is prescribed. Intravaginal dehydroepiandrosterone (DHEA), an androgen derivative, has been evaluated for treating GSM and research is ongoing. ${ }^{4}$

It can, therefore, be concluded that GSM is indeed a neglected condition unrecognized by the women and often underdiagnosed by the physicians. Health education seminars as well as leaflets for the women and updates for the caregivers are the need of the day and cannot be overemphasized.

\section{REFERENCES}

1. Harlow SD, Gass M, Hall JE, Lobo R, Maki P, Rebar RW, Sherman S, Sluss PM, deVilliers TJ. The straw +10 collaborative group. Executive summary of the stages of reproductive aging workshop +10 : addressing the unfinished agenda of staging reproductive aging. Climacteric 2012;15(2):105-114.

2. Nappi RE, Kokot-Kierepa M. Vaginal Health: insights, views and attitudes (VIVA) results from an international survey. Climacteric 2012;15(1):36-44.

3. Portman DJ, Margery LS, Gass MD. Menopause. The Journal of The North American Menopause Society 2014;21(10).

4. Management of symptomatic vulvovaginal atrophy: 2013 Position Statement of The North American Menopause Society. Menopause 2013;20(9):888-902.

Syeda Batool Mazhar FRCOG (UK) FCPS (PK) MCH Centre, PIMS, SZABMU Islamabad, Pakistan 